\title{
Correction to: An air quality prediction model based on CNN-BiNLSTM-Attention
}

\author{
Jingyang Wang ${ }^{1}$. Jiazheng $\mathrm{Li}^{1} \cdot$ Xiaoxiao Wang ${ }^{1}$. Tingting Wang ${ }^{1}$ - Qiuhong Sun ${ }^{1}$
}

(C) Springer Nature B.V. 2022

\section{Correction to: Environment, Development and Sustainability https://doi.org/10.1007/s10668-021-02102-8}

Unfortunately, in the original publication of the article, the word "air" has not been included in the article title. The correct article title is "An air quality prediction model based on CNN-BiNLSTM-Attention".

The original article has been corrected.

Publisher's Note Springer Nature remains neutral with regard to jurisdictional claims in published maps and institutional affiliations.

The original article can be found online at https://doi.org/10.1007/s10668-021-02102-8.

Qiuhong Sun

sunqiuhong@hebust.edu.cn

Jingyang Wang

jingyangw@hebust.edu.cn

Jiazheng Li

69880353@qq.com

Xiaoxiao Wang

1772732517@qq.com

Tingting Wang

3509922875@qq.com

1 School of Information Science and Engineering, Hebei University of Science and Technology, Shijiazhuang 050018, China 